\section{P173 ROUTINE ENQUIRY FOR INTIMATE PARTNER VIOLENCE (IPV) ACROSS AN INTEGRATING SEXUAL HEALTH SERVICE}

Janani Jaganathan, Christine Donohue, Sophie Brady*, Nicola Fearnley. Bradford Teaching Hospitals NHS Foundation Trust, Bradford, West Yorkshire, UK

\subsection{6/sextrans-2015-052126.216}

Background We have previously presented our review of routine enquiry (RE) for IPV in a genitourinary medicine (GUM) service. On-going integration with contraception services (CASH) combined with a new electronic patient record (EPR) in 2013 has prompted further review across the whole service (comprising 13 community clinics and the level 3 GUM service).

Aim Have these service changes impacted on our recommendation that RE is undertaken for all new patients? In addition, how many cases of IPV are we identifying?

Methods All new or rebook patients attending between 01/05 and 30/11/2014 where RE was documented were reviewed. Results: There were 17878 attendances (12316 new; 8724 female, 3590 male). The results are summarised below.

\begin{tabular}{lll} 
Abstract P173 Table 1 & Routine enquiry for intimate violence \\
\hline IPV routine enquiry & No of cases & $\%$ \\
\hline Patient screened at least once & 8614 & 70 (of new attendances) \\
Current issues documented & 72 (68 female; 4 male) & 0.8 \\
Past issues documented & 567 & 6.6 \\
\hline
\end{tabular}

$58 \%$ of those identified with current issues of IPV had attended the level 3 GUM service. In the majority, support was already in place. 567 had documentation of past issues of IPV, of which 58 had on-going needs identified. Experiences included child sexual abuse, stalking and social media harassment.

Discussion Routine enquiry for IPV is feasible across an integrated service and identifies a range of issues. The proportion screened appears stable (71\% in 2013 and $70 \%$ in 2014). The scale of the problem in our population is alarming and highlights the need for adequate staff training and clear referral pathways.

\section{P174 IMPROVING IMPLANT RETENTION RATES IN AN INTEGRATED SEXUAL HEALTH SERVICE}

Belinda Loftus*, Nicola Fearnley, Sophie Brady. Bradford Teaching Hospitals NHS Foundation Trust, Bradford, West Yorkshire, UK

\subsection{6/sextrans-2015-052126.217}

Background Subdermal implants (SDI) are cost effective when used for the recommended time. Early removal of SDI reduces cost effectiveness and we were aware anecdotally that this was an issue within our service. Data was lacking however. Subsequently removal rates at 3 and 6 months have been included within our Public Health Quality Contract (PHQC). Various strategies were implemented to reduce early removal. These included: improving the consent process by amending consent form; encouraging the use of additional methods to manage unscheduled bleeding; starting a dedicated implant removal clinic in February 2014.

Aim Have the outlined service changes impacted on SDI removal rates?

Methods Data from our PHQC was obtained from 2014-15. This measured SDI removal rates as a proportion of the total SDI fitted by our service.
Results In April 2014 the 3 months removal rate was 3.53\%. By November 2014 it had fallen to $0.34 \%$.

Discussion The strategies that were implemented appear to have had the desired effect. Care was taken to ensure staff gave patients the right information prior to fitting to ensure that their expectations of how any side effects would be managed was clear at the outset. The implant removal clinics were initially slow to get established and now are fully booked for months in advance. This has led to some criticism that patients are now unable to get their implants removed easily. The challenge moving forward is to ensure that patients have any symptoms managed promptly whilst keeping retention rates high.

\section{P175 THE IMPACT OF INTRODUCING AN ASSISTANT PRACTITIONER TO THE HEALTH ADVISOR TEAM IN A BUSY URBAN SEXUAL HEALTH SERVICE}

Belinda Loftus*, Nicola Fearnley, Sophie Brady. Bradford Teaching Hospitals NHS Foundation Trust, Bradford, West Yorkshire, UK

\subsection{6/sextrans-2015-052126.218}

Background The role of Assistant Practitioner (AP) in the Health Advisor (HA) team is new and has not previously been described. It is unusual in that someone who has not had formal nursing or medical training is able to supply medicines to a patient. Our service set about developing this Agenda for Change band 4 role, with Drug and Therapeutic Committee approval, to support the clinical team.

Aim To evaluate the new role now that training is complete.

Methods List the tasks undertaken by HA and compare with those now undertaken by the AP.

Results

\begin{tabular}{lc}
\hline Traditional HA Roles & Delegated to AP \\
\hline Supply Azithromycin for uncomplicated Chlamydia/contacts of & $\checkmark$ \\
(including in pregnancy) & \\
Carrying out simple Partner Notification (PN) for Chlamydia & $\checkmark$ \\
Recalling patients for treatment & $\checkmark$ \\
Following up patients via telephone calls & $\checkmark$ \\
HIV Point of Care Testing & $\checkmark$ \\
Motivational Interviewing & $\times$ \\
Carrying out PN for STls other than Chlamydia & $\times$ \\
Safeguarding Adults and Children & $\times$ \\
Support post Sexual Assault &
\end{tabular}

Discussion The AP has proved to be a valuable additional role within our service. Rigorous training and robust protocols had to be developed but this now allows her to operate independently. Despite initial reservations about replacing a HA post with an AP of a lower band our clinical team are now supportive of this role and recognise that this delegation of tasks allows time to focus on more complex cases.

\section{P176 THE ROLE OF DUTY DOCTOR IN AN INTEGRATED SEXUAL HEALTH SERVICES}

Laura Percy ${ }^{*}$, Mayur Chauhan. New Croft Centre, Newcastle Upon Tyne, UK

10.1136/sextrans-2015-052126.219 